\title{
The BLADE-ON-PETIOLE genes of Arabidopsis are essential for resistance induced by methyl jasmonate
}

\author{
Juan Vicente Canet ${ }^{1}$, Albor Dobón ${ }^{1}$, Jana Fajmonová ${ }^{2}$ and Pablo Tornero ${ }^{3^{*}}$
}

\begin{abstract}
Background: NPR1 is a gene of Arabidopsis thaliana required for the perception of salicylic acid. This perception triggers a defense response and negatively regulates the perception of jasmonates. Surprisingly, the application of methyl jasmonate also induces resistance, and NPR1 is also suspected to be relevant. Since an allelic series of npr 1 was recently described, the behavior of these alleles was tested in response to methyl jasmonate.

Results: The response to methyl jasmonate of different npr1s alleles and NPR1 paralogs null mutants was measured by the growth of a pathogen. We have also tested the subcellular localization of some npr1s, along with the protein-protein interactions that can be measured in yeast. The localization of the protein in npr 1 alleles does not affect the response to methyl jasmonate. In fact, NPR1 is not required. The genes that are required in a redundant fashion are the BOPs. The BOPs are paralogs of NPR1, and they physically interact with the TGA family of transcription factors.

Conclusions: Some npr 1 alleles have a phenotype in this response likely because they are affecting the interaction between BOPs and TGAs, and these two families of proteins are responsible for the resistance induced by methyl jasmonate in wild type plants.
\end{abstract}

Keywords: Methyl jasmonate, Salicylic acid, Arabidopsis, NPR1, BOPs, Defense

\section{Background}

Plants are constantly defending themselves against pathogens by means of a wide array of mechanisms. Some of them are pre-existing (or non inducible) and others are induced in response to the pathogen attack. Salicylic acid (SA, reviewed by [1]) is a plant hormone which is crucial for the inducible response of Arabidopsis thaliana (Arabidopsis) to biotrophic pathogens like Pseudomonas spp [2]. When a pathogen is perceived, SA is produced and accumulated, producing a proper defense. This SA signaling occurs not only where the attack takes place, since defense is also enhanced in leaves different from the one inoculated. This is called Systemic

\footnotetext{
* Correspondence: ptornero@ibmcp.upv.es

${ }^{3}$ Instituto de Biología Molecular y Celular de Plantas (IBMCP), Universidad Politécnica de Valencia (UPV)-Consejo Superior de Investigaciones Científicas (CSIC), Ciudad Politécnica de la Innovación (CPI), Ed. 8E; C/ Ingeniero Fausto Elio s/n, Valencia 46022, Spain

Full list of author information is available at the end of the article
}

Acquired Resistance (SAR, [3]). SA has an intricate crosstalk with other hormones, showing an overall negative crosstalk with auxins, ethylene (ET), and jasmonates (JA, crosstalk of hormones reviewed by [4]). In the case of JA, it has been shown that the active form in planta is JA-Ile (reviewed by [5]), while in the laboratory is used exogenously as Methyl Jasmonate (MeJA).

NON-EXPRESSOR OF PATHOGENESIS-RELATED GENES1 (NPR1) is the main gene required for SA perception [6]. There are five paralogs of NPR1 in Arabidopsis [7], BLADE-ON-PETIOLE1 (BOP1) and BOP2 have an important role in development [8], NPR3 and NPR4 have a role in defense [9], probably through SA perception [10], and no specific function for NPR2 has been described, besides a secondary role in SA perception [11]. There are other genes that are relevant for signal transduction, like the family of TGA transcription factors whose products interact with NPR1 [12], but they are required in a redundant fashion. 
NPR1 has been described as having more than one role in defense, since it is also important in the Induced Systemic Resistance (ISR, [13]). ISR is defined as the resistance triggered at the leaves by a non pathogenic organism inoculated in the roots, and while SAR requires SA signaling, ISR requires MeJA and ET signaling. As with SA, exogenous applications of MeJA and ET trigger resistance in Arabidopsis towards some biotrophs, like Pseudomonas [14]. It has been proposed that NPR1 is relevant for the resistance-inducing ability of MeJA ([13], hereafter abbreviated as RIM), although RIM it is not necessarily equivalent to ISR. While the role of NPR1 in SA perception takes place in the nucleus [15], its function in RIM is not so clearly understood. It has been described a cytosolic function of NPR1 crucial in cross-talk between SA and JA signaling [16]. Furthermore, Arabidopsis transcriptome analysis upon pathogen infection has suggested that such cytosolic function is also involved in the modulation of JA-dependent defenses [17]. The npr1-3 mutant, which produces a truncated cytoplasmatically localized npr1 protein with no nuclear localization signal, has been reported to be affected only in SA-dependent gene expression, not in JA and ET dependent genes. In contrast, the npr 1-1 mutant, which has a mutation in a key domain, is affected in the expression of SA, JA and ET-dependent genes [17]. More recent studies support such cytosolic NPR1 function as regulator of JA-dependent defense responses $([18-19,20])$.

BOP1 was first identified by its mutant phenotype of ectopic blades along the petioles, as well as some alterations in the flowers [8]. The first allele identified was a dominant negative, since T-DNA insertions in bop1 did not reproduce the phenotypes of ectopic blades [21]. Once BOP1 was identified as paralog to NPR1 [22], it was shown that other paralog, $B O P 2$, functions redundantly with BOP1 [21]. The double mutant bop1 bop2 reproduced all the developmental phenotypes of ectopic blades, but it was wild type when inoculated with Pseudomonas [21], and it is also wild type for SA perception [23].

Since a collection of npr 1 alleles has recently been available [11], we tested the hypothesis that the role of NPR1 in RIM is cytosolic. In this work, we show that NPR1 has no role in RIM in wild type conditions, since the genes responsible for RIM are BOP1 and BOP2, with an important part being played by the TGAs. Therefore, two genes required for the normal development of the leaf, are also required for plant defense.

\section{Results}

Role of NPR1 in RIM

NPR1 has been characterized as a result of observing the response to SA of the great number of alleles described for it [24]. NPR1 has also been described as essential for RIM, but there are differences between alleles, since npr1-1 and npr1-3 have different RIM ([20,25]). npr1-1 and npr1-3 have other differences in phenotypes related to MeJA. Thus, the SA-JA antagonism is not present in $n p r 1-1$, but it is active in npr1-3 [16]. Other difference is the gene expression, whereas nprl-1 was affected in SA, JA, and ET dependent genes upon Pto inoculation, npr 1-3 was only affected in SA dependent genes [17]. These different phenotypes have been attributed to the lack of nuclear localization in npr1-3, since the truncated cytosolic protein would be functional to modulated JA-dependent defense response [19].

In order to determine the precise role of NPR 1 in RIM, and to asses the role of the cytosolic vs. nuclear localization, we tested an allelic series of 43 npr 1 alleles, defined by their inability to perceive SA [11]. The npr1 alleles produced a mixture of phenotypes in RIM (Figure 1), and out of the 43 alleles tested, 11 had a significant RIM (RIM+, Figure 1a), meaning that the growth of Pto in MeJA treated plants was significantly lower than in mock treated plants (Student's test of one tail, with $\mathrm{P}<0.05)$. The rest of alleles showed no response (RIM-, 11 out of 32 are shown in Figure 1b). These two categories of alleles did not share any obvious feature and had the same proportion of stops and point mutations in each category. In fact, in the RIM+ alleles tested, six point mutations were widely scattered along the protein (Figure 1c, npr1-3 is included as reference).

These alleles could somehow affect the localization of the protein inside the cell, even if the mutation was not in the NLS. To check this possibility, cDNAs of three RIM+ alleles (npr1-22, -35 and -44, Additional file 1), and three RIM- alleles (npr1-1, -40, and -56, Additional file 1), chosen among the point mutations, were cloned along with the wild type NPR1. Then, these seven cDNAs were transiently expressed in $N$. benthamiana under the control of the $35 \mathrm{~S}$ promoter and with the GFP marker. The free GFP was detectable in the nucleus and in the cytoplasm. But when the wild type and the six alleles of npr1 were expressed in the same conditions, GFP was detected mainly in the nucleus, with no difference existing between the two classes of alleles (Figure 2a).

As a complementary approach, we took advantage of the transgenic line that overexpresses NPR1 fused to the steroid hormone binding domain of the rat glucocorticoid receptor $(\mathrm{HBD}$, and the transgenic plants are known as NPR1-HBD, [15]). NPR1-HBD remains exclusively in the cytosol in mock conditions and should be functional in RIM. The original line is in an npr1-3 background $(\mathrm{RIM}+)$, and therefore the transgene was transferred to an npr1-1 background (RIM-) to check for complementation. Treatments with BTH and with and without 


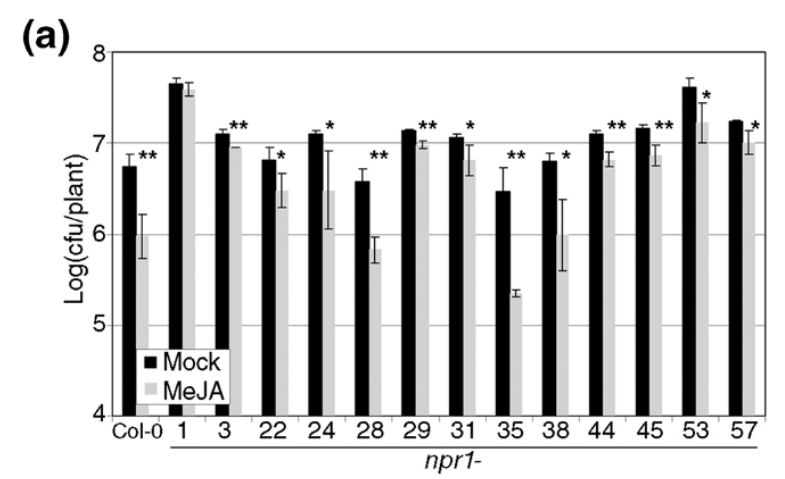

(b)

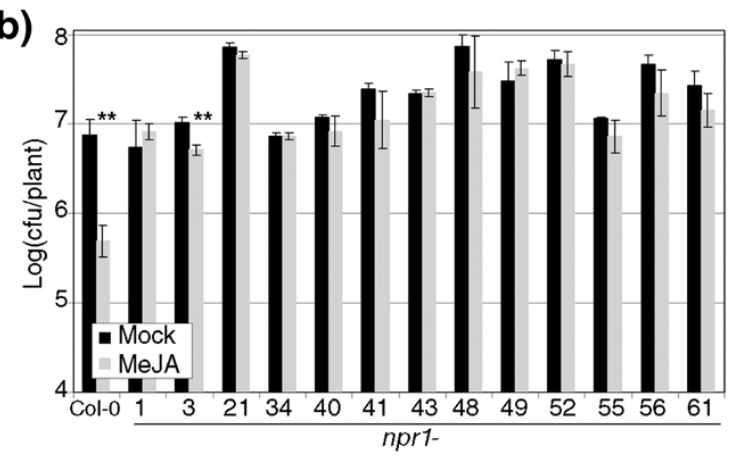

(c)

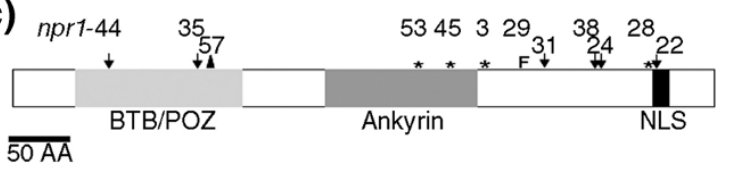

Figure 1 npr 1 alleles differ in their response to MeJA. Fortythree $n p r 1$ alleles were treated with $100 \mu \mathrm{M}$ methyl jasmonate (MeJA), with $0.1 \%$ DMSO and $0.02 \%$ Silwet L-77 or a mock treatment. One day later, Pseudomonas syringae pv. tomato DC3000 (Pto) was inoculated and its growth measured three days later in a logarithmic scale. For each genotype and treatment three samples with 5 plants per sample were taken. The bars show the average \pm SD and npr $1-1$ and npr $1-3$ are included as controls for negative and positive response to MeJA respectively. (a) npr1 alleles that show resistance induced by MeJA. (b) nprl alleles that do not show resistance induced by MeJA. (c) Schematic representation of the npr1 alleles that showed resistance induced by MeJA along the structure of NPR1. BTB/POZ stands for Broad-Complex, Tramtrack and Bric-a-brac proteins, Pox virus and Żinc finger proteins. Ankyrin for Ankyrin Repeat Motifs (4 of them) and NLS for Nuclear Localization Signal. The arrows indicate point mutations, the asterisks stop codons, the letter " $\mathrm{F}$ " frameshift, and a triangle an internal deletion. The number indicates the number of allele. In all figures, the experiments were repeated at least three times with similar results. One asterisk means a significant difference with $P<0.05$, and two asterisks means $\mathrm{P}<0.01$ (Student's test of one tail).

glucocorticoid dexamethasone (DEX) showed that NPR1-HBD was functional (Additional file 1). NPR1HBD, even under the control of the $35 \mathrm{~S}$ promoter, did not complement the lack of RIM in npr1-1 (Figure 2b). When DEX was applied, NPR1-HBD moved to the nucleus and nprl-1 was complemented in the RIM phenotype. Note that the presence of cytosolic NPR1HBD in an npr1-3 background did not enhance RIM in comparison to npr1-3 alone.

The npr1 alleles RIM+ or RIM- did not share any obvious feature, so it would be difficult to assign a precise role to the wild type gene. A critical genetic resource to discern the role of a gene is the null allele. Therefore, the response of two null alleles of $n p r 1$ to RIM was measured (Figure 3a). Both npr1-70 and npr1-71 are in Laer-0 background, so an introgression of npr1-1 in Laer-0 was used as control [11]. These two null alleles responded to RIM in all experiments like the wild type. npr1-70 introgressed in Col-0 responded again like the wild type, which ruled out any ecotype effect (Figure $3 b$ ). Since the direct role of NPR1 in RIM was in question as a consequence of the aforementioned results, we included an independent RIM- control, coi1-40 (see Methods).

The role of NPR1 in this response might be indirect. Thus, one scenario would be a reinforcement of the negative crosstalking between SA and MeJA. npr 1 alleles produced more SA when infected with Pto [26] and seemed unable to metabolize it [27]. RIM- alleles defective in terms of SA perception- might have left intact the negative crosstalk between SA and MeJA, and an excess of SA repressed the response to MeJA beyond the wild type levels. Therefore, the RIM+ alleles would be defective in terms of both SA perception and SAMeJA crosstalking, an explanation that would also be in agreement with the behavior of the null alleles.

To test this hypothesis, the double mutants between npr1-1 and NahG (a transgenic plant that degrades SA, [28]), eds5 (a mutant in SA transport, [29]), and sid2 (a mutant in SA biosynthesis, [30]), were constructed and tested for RIM. nprl-1 did not respond to MeJA even if the levels of SA were low (Figure 3c), so the hypothesis of a reinforcement of the negative crosstalk was not supported.

\section{$B O P 1$ and $B O P 2$ and their role in RIM}

The experiments with the null alleles showed that NPR1 was not necessary for RIM. Perhaps NPR1 and other gene(s) would be redundantly responsible of RIM, and while null npr1 alleles would have a RIM+ phenotype, some npr1 alleles could be RIM- by interacting with other protein(s) negatively. The most likely candidates for these interactions were the NPR1 paralogs, since their proteins shared the same overall structure. There are five additional paralogs of NPR1 in the Arabidopsis genome [7], and we analyzed double mutants of npr1-70 with different paralogs (Figure 4). There was no proof of NPR1 having a redundant role in RIM. NPR2 did not play a significant role in RIM (Figure 4a) and the same was true for the double npr3 npr4 (Figure 4b,c and [7]). Strikingly, the double bop1 bop2 [21] was RIM- (Figure 4d). 

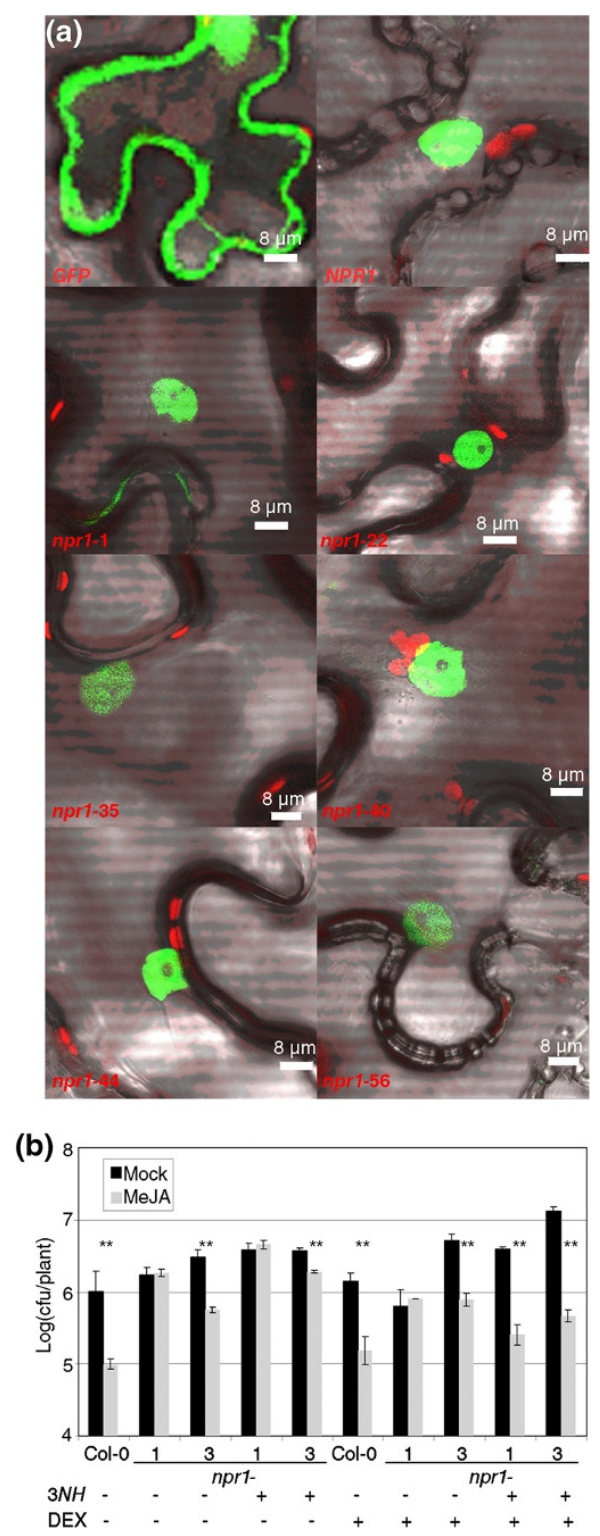

Figure 2 The nuclear localization of $n p r 1$ alleles is not relevant for resistance induced by MeJA. (a) Transient expression of GFP, NPR1:GFP, and six npr1 alleles (three from Figure 1a and three from Figure 1b). Agrobacterium tumefaciens containing the mentioned genes under the promoter 355 were infiltrated in leaves of Nicotiana benthamiana, and the expression was detected with a confocal microscopy four days later. (b) The cytoplasmic anchoring of NPR1 does not complement npr1-1 in its response to MeJA. Plants with the transgene 35SCaMVp:NPR1:HBD (abbreviated as 3NH) in npr1-1 or npr 1-3 background and its controls were treated with either dexamethasone (DEX) or mock solution, and then treated with either MeJA or mock solution. One day later, Pto was inoculated and measured as described in Figure 1. One asterisk means a significant difference with $\mathrm{P}<0.05$, and two asterisks means $\mathrm{P}<0.01$ (Student's test of one tail).
Single bop1 and bop2 were also tested for RIM and showed to be wild type (Figure 5a). BOP1 and BOP2 exert their function in part through transcriptional regulation of KNAT6 and physical interaction with PAN (TGA8, [31]), but T-DNA insertions predicted to disrupt KNAT6 or PAN activity did not have an effect on RIM (Figure $5 \mathrm{a}$ ). To rule out the possibility that other mutations besides bop 1 and bop 2 were producing this RIMphenotype, we constructed an artificial microRNA (amiRNA, [32]) to deplete the levels of BOP1 and BOP2 at the same time. Eight independent homozygous transgenic lines for amiRNA (BOP1 - BOP2) were analyzed for RIM (Figure $5 \mathrm{~b}$ ). Five out of eight lines were RIM-, and the remaining three responded less than the wild type control. The levels of both genes were partially depleted in the eight lines (Figure $5 \mathrm{c}$ ); five of the lines had both genes significantly reduced, and all had BOP2 significantly reduced. None of these lines had the characteristic blade-on-petiole macroscopic phenotype, not even as the subtle phenotype of bop1 alone.

The previous experiments had shown that BOP1 and $B O P 2$ were acting redundantly in RIM (Figure $4 \mathrm{~d}$ ). Therefore, increasing the amount of any of them should have an effect on RIM, especially since normal levels of $B O P 1$ and $B O P 2$ are quite low (Additional file 1, [21]). The overexpression lines of $B O P 1$ and $B O P 2$ described [33] were analyzed for RIM. 35S:BOP1 had a stronger RIM than Col-0, and 35S:BOP2 had a strong variation in the MeJA treated plants (Figure 6a). At the time of the experiments, each population looked homogeneous, but when these plants were grown to set seeds, two phenotypes could be observed in each transgenic line. Approximately half of the plants showed a wild type phenotype, and the other half reproduced the dwarf plants described [33]. Seeds from both lines and from both phenotypes reproduced the two phenotypes. It seems that it was an issue of silencing, since RNA taken from plants classified by their mentioned phenotype diverged widely in the transgene expression (Figure 6b).

\section{bop1 bop2 specificity in RIM}

The bop 1 bop 2 double mutant lacked a RIM response, but this failure in MeJA signaling might occur at different points of the signal transduction. For example, the defect could target a general signaling component affecting all MeJA responses (e.g. coil, [34]), or a specialized part of the pathway, affecting a subset of MeJA responses (e.g. jin1, [35]). When bop1 bop2 plants were grown in plates containing MeJA, the growth of the roots was similar to the wild type controls (Figure 7a). Other phenotypes of bop1 bop2 plants growing in MeJA plates were similar to the wild type controls (carotenoids production, size of aerial part, number of trichomes, etc.; data not shown). Another effect of MeJA is the 


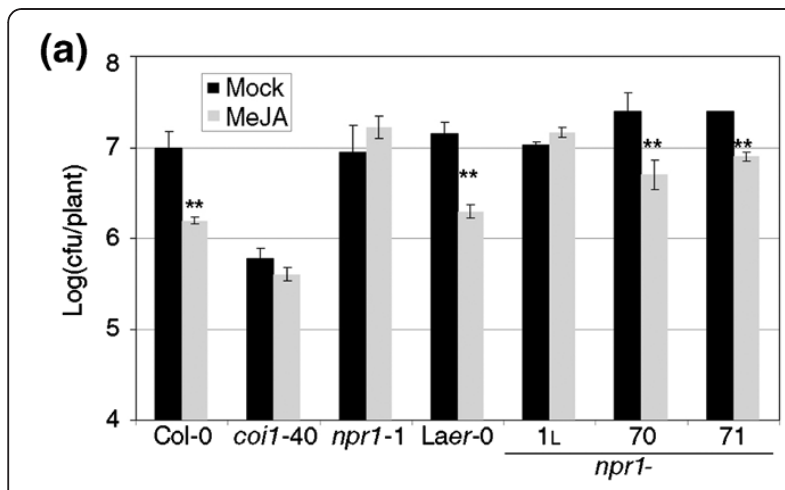

(b)
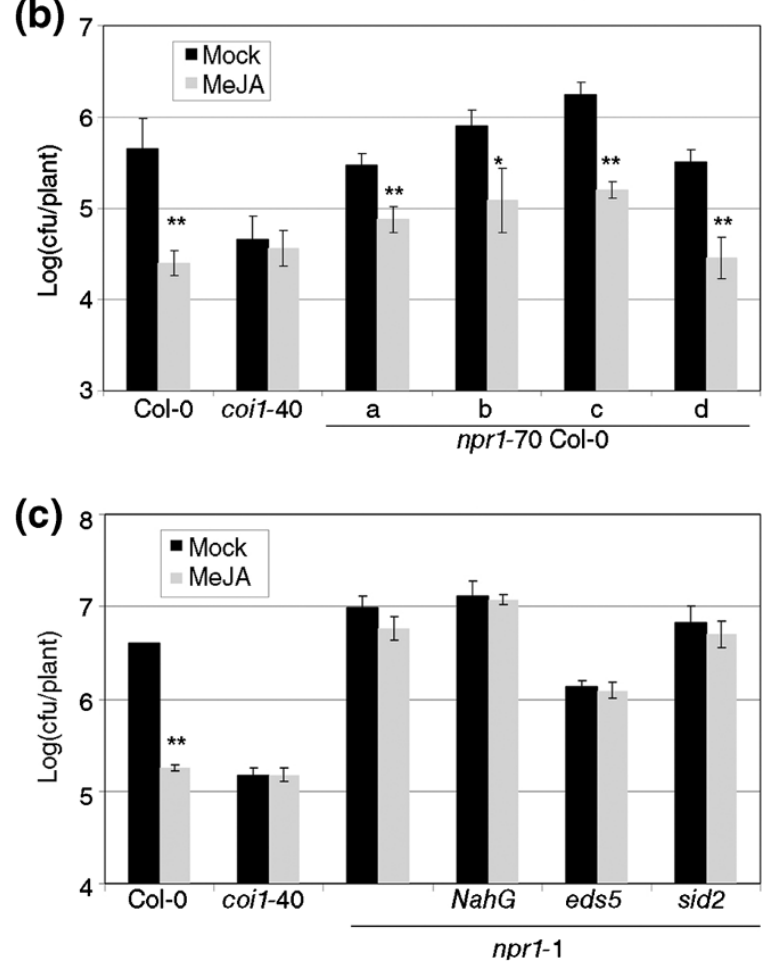

Figure 3 NPR1 is not necessary for resistance triggered by MeJA. (a) Two null npr1 alleles (npr1-70 and npr1-71, both in Laer-0 background), plus their controls were tested for resistance induced by MeJA as described in Figure 1. coil-40 is introduced as negative control for resistance triggered by MeJA. $n p r 1-1_{L}$ is $n p r 1-1$

introgressed in Laer-0 [1 1]. (b) Introgressed lines of npr1-70 in Col-0 show the same phenotype than the original npr1-70. (c) The effect of some npr1 alleles on resistance triggered by MeJA is not due to an excess of salicylic acid. Double mutants of npr1-1 with NahG, eds5, and sid2 were tested for their response to MeJA in resistance. One asterisk means a significant difference with $\mathrm{P}<0.05$, and two asterisks means $P<0.01$ (Student's test of one tail).

increase of senescence in detached leaves, measured as chlorophyll production [36]. bop1 bop 2 responded as the wild type in this particular system (Figure 7b). Coronatine is a virulence factor of several Pseudomonas isolates with structural and functional similarities to JA-Ile (the functional form of Jasmonate in planta, [5]), therefore a mutant Pto that lacks coronatine [37] grows less in Col-0 than the wild type Pto. bop 1 bop 2 was also wild type in response to Pto with and without coronatine (Figure 7c). Inoculations with Plectospharaella cucumerina, a fungus that causes more disease in MeJA mutants than in wild type plants [38], did not cause any more disease in bop1 bop 2 than in Col-0 (data not shown). If bop1 bop2 was not a MeJA signaling mutant, but specifically in RIM, it could be defective in the other signaling required for ISR; ET. It was not; when $1 \mathrm{mM}$ of 1 -aminocyclopropane1-carboxylic acid (ACC, an ET precursor) was sprayed to bop1 bop2, the resistance triggered was similar to that triggered in the wild type controls (Figure 7d). etr1 (ETHYLENE RESPONSE 1, [39]) was included as a negative control of resistance induced by ethylene.

\section{NPR1 and BOPs interactions}

Once it was clear that both BOP genes are required for RIM, we tested the model that npr1 RIM- alleles could have a dominant negative effect on BOP activity, either directly or indirectly. To first test whether NPR1 had an effect in the interaction between BOP proteins, we used a yeast two-hybrid assay. As reported, BOP1 and BOP2 interacted with each other [40]. Next, we introduced in a third plasmid containing wt NPR1 or various mutant $n p r 1$ alleles presented in Figure 2a. If the effect of npr1 on RIM were a direct interaction between NPR1 and the two BOPs, the alleles that diverge in their RIM phenotype would diverge in their ability to interfere in the interaction of BOP1 and BOP2. The two classes of npr 1 alleles did not have a distinct behavior (Figure 8a, the first three npr 1 alleles are RIM-, and the last three are $\mathrm{RIM}+$ ), therefore the dominant negative effect did not seem to be direct.

All the NPR1 paralogs tested interact with members of the TGA family in a different degree [7,21]. Therefore, the TGAs would be a reasonable candidate for being the third component, and their interaction with RIM- alleles would indirectly affect the function of BOP1 and BOP2. As a control, single mutants in TGA1 and TGA7 produced a significant RIM (Figure 8b), but when three specific tgas are knocked out at the same time (a triple which phenocopies an npr1 mutant in SA response, [41]), there is no RIM (Figure 8b).

We reasoned that one or several of these three TGAs (TGA2, 5, and 6) might have a functional interaction with the BOPs, which might be affected by the RIMalleles. To test this hypothesis BOP1 and each of the mentioned TGAs were introduced in the yeast twohybrid system with the npr 1 alleles mentioned above in a third plasmid. TGA2 and TGA6 interact differentially with BOP1 depending on the npr1 protein present (Figure 8c). There was an enhancement of the interaction in two out of the three RIM- alleles, and no 
(a)

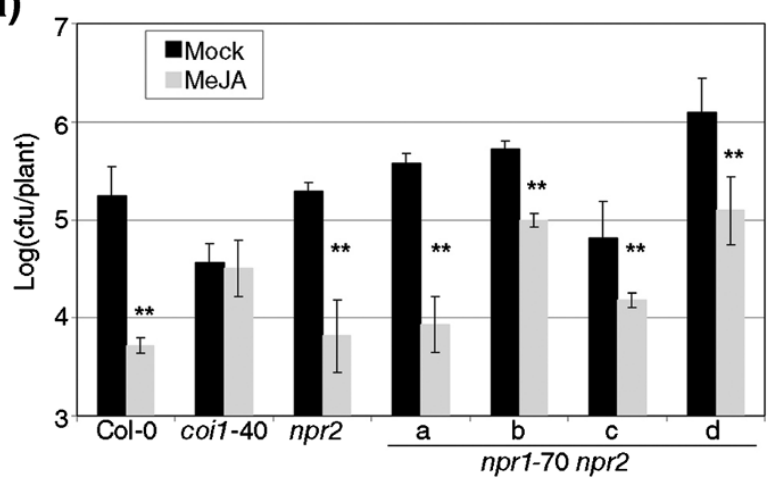

(c)

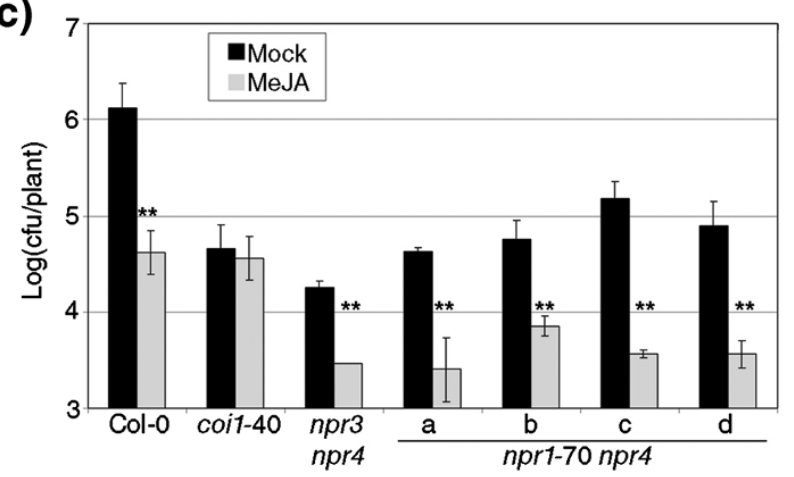

(b)

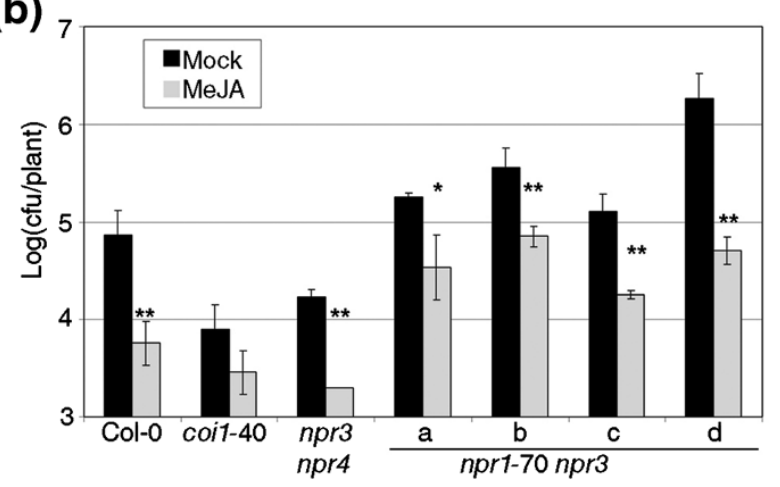

(d)

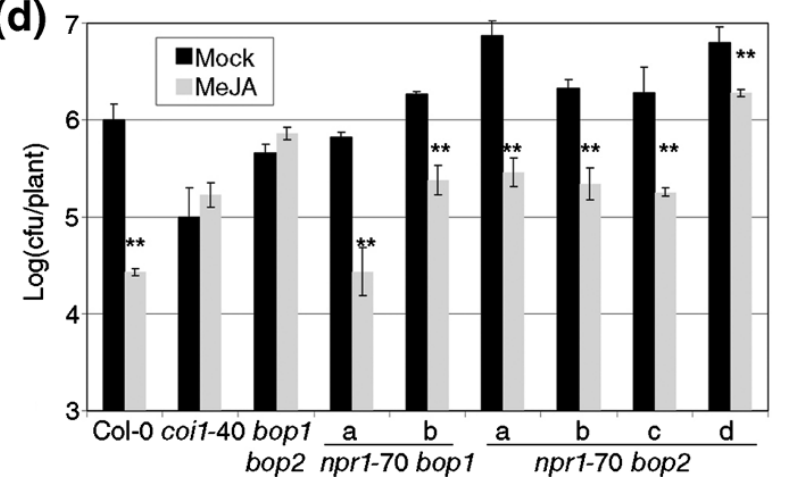

Figure 4 A role for the NPR1 paralogs in resistance triggered by MeJA. Double mutants of npr1-70 with NPR1 paralogs and their corresponding controls were tested for resistance triggered by MeJA as described in Figure 1. (a) Double mutants with npr2. (b) npr3. (c) npr4. (d) bop1 and bop2. Four independent lines were tested in each case, except with bop 1, where only two were obtained. One asterisk means a significant difference with $\mathrm{P}<0.05$, and two asterisks means $\mathrm{P}<0.01$ (Student's test of one tail).

interference in two out of the three RIM+ alleles. The interaction TGA5-BOP1 was not affected by the presence of npr1 proteins (data not shown). The experiments were repeated with BOP2 producing similar results (data not shown). In sum, the data indicated that BOPs and TGA2, TGA5 and TGA6 are required for RIM, that BOPs interact with these (and other) TGAs, and that NPR1 may modulate the affinity or stability of the interactions.

\section{Discussion}

\section{NPR1 is not required for RIM}

NPR1 is an essential gene for SAR and SA perception [6]. npr1-1, the most widely used allele, is also impaired in RIM [13]. We speculated that since npr1-3 is wild type for RIM $([20,25])$, and it has been reported that the difference of some phenotypes between npr1-1 and $n p r 1-3$ was due to the lack of NLS in npr1-3 ([17,19]), the same could be true for RIM. However, we show here that the nuclear localization of the alleles makes no difference. This conclusion is supported by multiple lines of evidence. First, the nprl alleles with RIM+ are not structurally similar to npr1-3, since not all of them are affected in the NLS (Figure 1c). Even an allele with a point mutation in the NLS (npr1-22, Additional file 1) should be partially localized in the nucleus [15]. Second, three RIM- and three RIM+ alleles do not differ in their nuclear localization or stability when transiently expressed in $N$. benthamiana (Figure 2a, Additional file 1). While these proteins are no longer functional, they respond to the signals of a wild type background by localizing in the nucleus. Third, when a functional NPR1 is anchored in the cytoplasm there is no complementation of the RIM- phenotype in an npr1-1 background (Figure 2b), nor there is an increase in RIM phenotype in an npr1-3 background. In fact, the application of DEX triggered an increased RIM in both backgrounds (discussed below).

But, most importantly, NPR1 is not required for RIM, since the null npr1 alleles are RIM+ regardless of the background (Figure 3a,b). We also discarded that NPR1 could be a part of RIM in a redundant fashion with its paralogs (Figure 4).

An interesting alternative for the role of NPR1 in RIM would be an effect on the crosstalking between SA and MeJA. NPR1 has been described as a key point in the negative regulation between SA and MeJA. Thus, the RIM+ alleles could be defective in both SA perception 


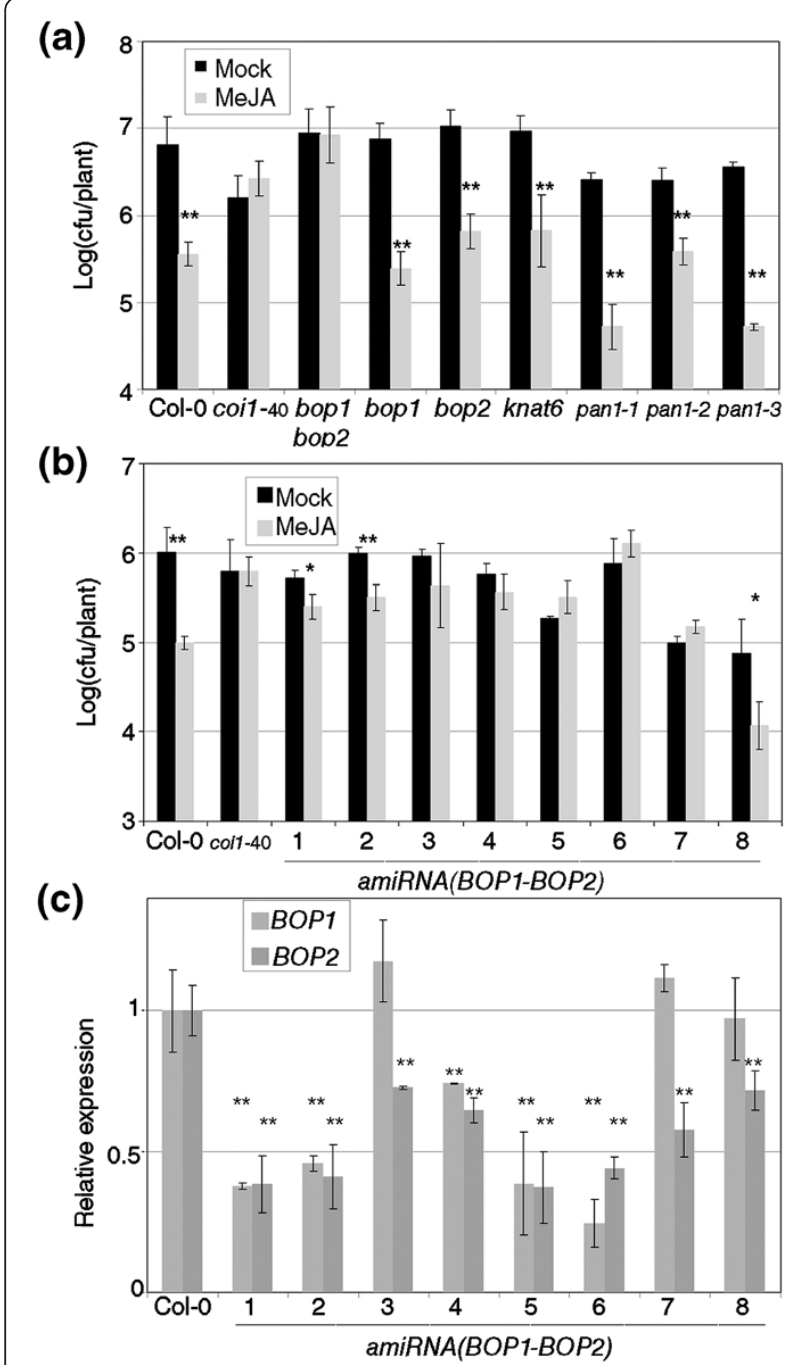

Figure 5 Only the reduction of both bop 1 and bop2 expression affects resistance triggered by MeJA. (a) The double bop1 bop2, the single mutants, T-DNAs insertions in KNAT6 and PAN1, and their controls were tested for resistance induced by MeJA as described in Figure 1. KNAT6 and PAN1 are genes that interact with BOP1 and BOP2. (b) Reduction of $B O P 1$ and $B O P 2$ expression partially phenocopies the double bop1 bop2. Eight independent transgenic lines of an artificial micro RNA designed to reduce the levels of BOP1 and BOP2 (amiRNA (BOP1-BOP2) were tested as described in Figure 1. The lines did not show any macroscopic blade-on-petiole phenotype. (c) RNA was extracted from 3-week-old plants of the lines described in (b), and transcript levels for BOP1 and BOP2 were measured by means of RTqPCR. Levels of expression are normalized to three reference genes and to the level of Col- 0 in mock. Asterisks mark the significance of the difference between the levels of expression of each line with Col-0; one asterisk means a significant difference with $P<0.05$, and two asterisks means $P<0.01$ (Student's test of one tail).

and in SA-MeJA crosstalk, while the RIM- alleles would be defective only in SA perception but not in SA-MeJA crosstalk. The inoculation with Pto triggers an increase in the levels of SA, and in the case of the npr1 alleles, there is more SA than in the wild type [42]. Although this hypothesis would explain the phenotype of the null alleles, it was rejected after the experiment of Figure 3c, where a severe reduction of SA levels in a RIM- allele did not have any effect on the phenotype.

\section{$B O P 1$ and $B O P 2$ are redundant in RIM}

The redundant functions of $B O P 1$ and $B O P 2$ are essential for normal development. Previous work has shown that the double mutant has numerous defects in plant architecture including altered leaf morphology [43], changes in floral patterning [21], defects in the conversion of shoots to flowers [44] and loss of floral-organ abscission [45]. The double mutant was tested for basal defense [21] and SA perception (Additional file 1) but no difference from wild type was found. We show here that both genes are also redundantly required in defense against pathogens triggered by MeJA. Interestingly, whereas significant loss of BOP activity is required to exert changes in development [21], RIM is abolished in plants that are only partially silenced for the BOP genes (Figure 5b,c). Thus, the levels of gene expression required for RIM are higher than those required for normal development. Compatible with this idea, BOPs expression in plants is highly localized, restricted to young organ primordia, leaf petioles, and lateral organ boundaries, which may make systemic responses to MeJA sensitive relatively minor changes in BOP transcript abundance. Both NPR1 and the BOPs localize to the cytoplasm as well as nucleus and interact with members of the TGA family of bZIP transcription factors, albeit with different affinities (e.g., [12]). In development, BOP1 and BOP2 form a nuclear complex with TGA8/ PERIANTHIA (PAN) to regulate number of sepals and petals in flowers and potentially to promote floral meristem fate [21]. Given that pan loss-of-function did not reproduce the RIM- phenotype (Figure 5a) other genes, perhaps TGAs, are involved in this phenotype, as shown for SA perception [23]. Given that BOPs play both positive and negative roles in transcriptional regulation of the KNOX (Knotted1-like homeobox) gene KNAT6 [46], we also tested if RIM was affected by knat6 loss-of-function, but again, no difference was observed (Figure 5a). This may reflect redundancy with other KNOX genes, or more likely, that BOP regulation of RIM is independent of KNAT6.

Whether bop1 bop 2 recapitulates or not all the phenotypes of the RIM- npr1 alleles (e.g. ISR, [13]; Verticilium resistance, [18]; resistance induced by Piriformospora indica, [47]; etc.) remains to be assessed. We did check that there were similar phenotypes in the specificity of response to MeJA as well as the fact that bop1 bop2 was wild type for the rest of MeJA phenotypes (Figure 7). But there were strong differences, since npr1-3 is affected in basal defense and SA perception while bop 1 bop 2 is wild type for both phenotypes [21]. 

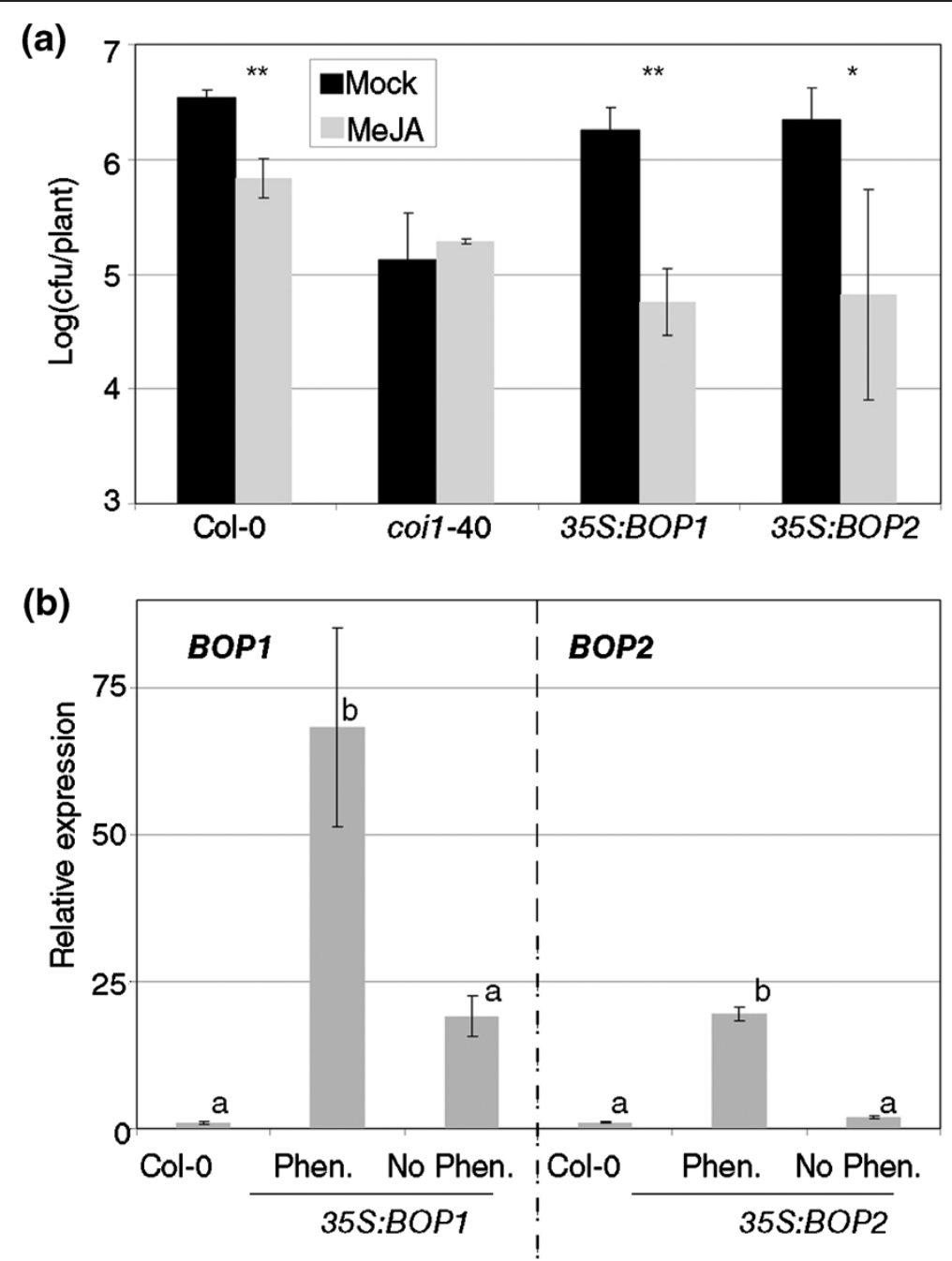

Figure 6 The overexpression of BOP1 or BOP2 produces more response to MeJA. (a) 35S:BOP1 and 35S:BOP2 lines [33] and their controls were tested as described in Figure 1. One asterisk means a significant difference with $P<0.05$, and two asterisks means $P<0.01$ (Student's test of one tail). (b) Silencing of the overexpression lines. 35S:BOP1 and 35S:BOP2 lines were phenotyped at the time of bolting as having a characteristic phenotype (Phen.) [33] or being wild type (No Phen.). Then, RNA was extracted from several plants and the levels of the transgenes quantified as described in Figure $5 \mathrm{c}$. On the left side of the plot, relative expression of BOP1 and, on the right, relative expression of BOP2. Means with the same letter are not significantly different (Fisher's LSD test, $\mathrm{P}<0.05$ ). The test was performed separately for each gene.

Regarding ET, the other hormone relevant for ISR, it has been proposed that applications of this hormone could render the crosstalk between SA and MeJA independent of NPR1 [19]. It seems plausible that the ET induced resistance works as the MeJA induced resistance and other proteins -perhaps NPR1 paralogs, but not the BOPs (Figure 7d) might also be affected by some alleles of $n p r 1$.

Some npr1 alleles interfere in the BOPs-TGAs interaction The RIM- npr1 alleles were the majority of the alleles found (32 RIM- vs. 11 RIM+). How is this compatible with the fact that the null nprl alleles are RIM+? A possible explanation was the selection used in the screening. Since the selection was made for complete loss of SA perception, perhaps most of the RIM+ alleles had a phenotype of partial SA perception, as the null alleles. Then, the prediction would be that a good number of random alleles of $n p r 1$ would be RIM+ and partially receptive to SA. We previously showed that for SA perception, there are genetic interactions between the npr1 alleles and the NPR1 paralogs [11]. The work reported herein points to a genetic interaction too, this being between npr1 alleles on one side and the BOPs on the other. Thus, the RIM- alleles were a phenocopy of the bop1 bop 2 mutant in defense but not in development. This discrimination was a consequence of the different thresholds for the phenotype in development and defense (Figure 5b,c).

Mechanistically, the levels of expression of the BOPs were low in comparison to NPR1 (Additional file 1), so a 

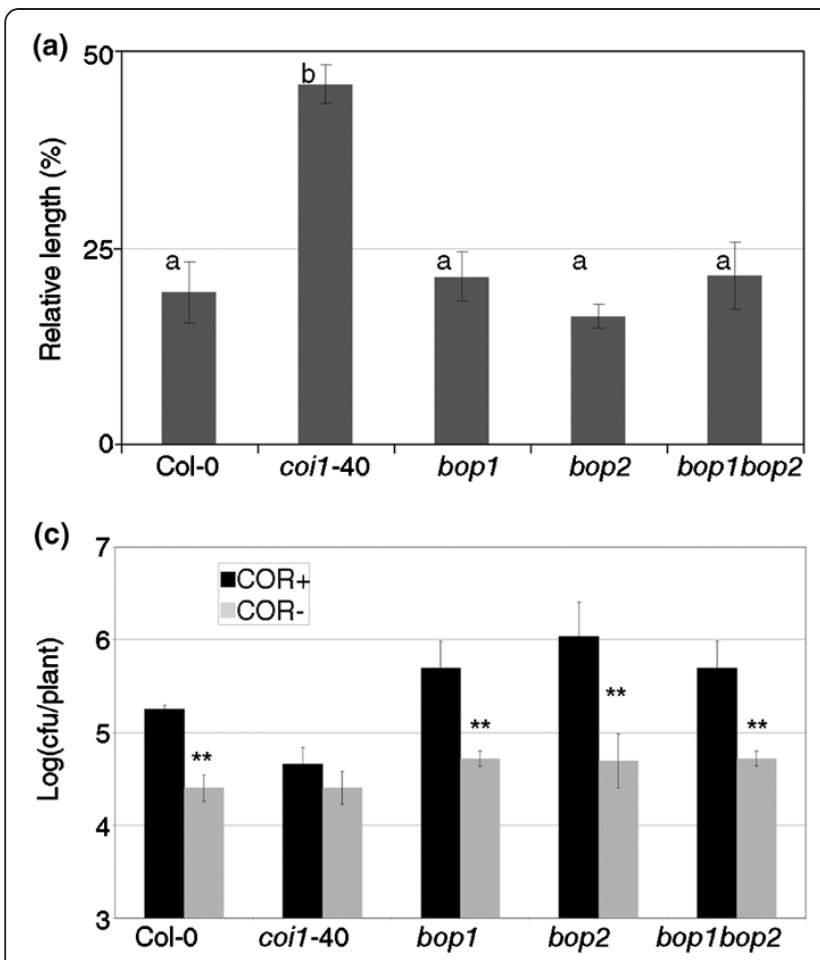
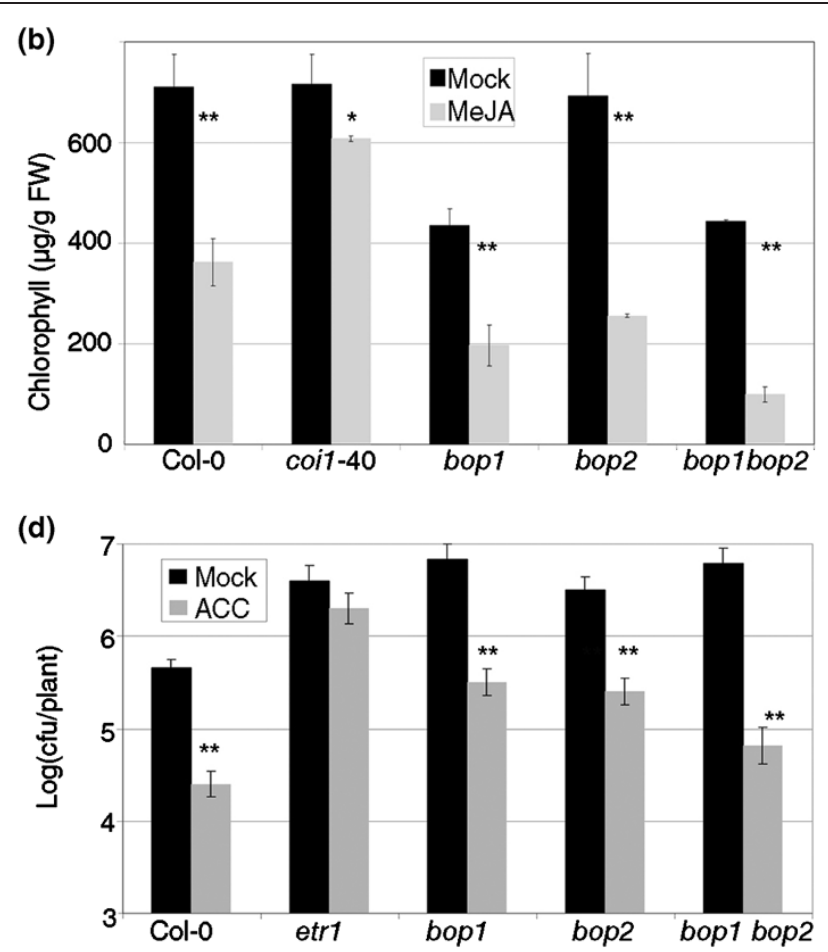

Figure 7 The double bop 1 bop 2 is specifically affected in the resistance induced by MeJA. (a) Length of primary root. bop 1 bop2 and their controls were grown in plates with Johnson's Media [58] with or without $50 \mu \mathrm{M}$ MeJA. At the age of 10 days, the lengths of the roots were measured in both conditions and their ratio (MeJA treated divided by mock treated) expressed as percentage. Means with the same letter are not significantly different (Fisher's LSD test, $\mathrm{P}<0.05$ ). (b) Senescence induced by MeJA. The indicated genotypes were grown in soil and mature leaves from 6-week-old plants were cut and floated on water with or without $100 \mu \mathrm{M}$ MeJA. The amount of chlorophyll (in $\mu \mathrm{g} / \mathrm{g}$ fresh weight) was measured after four days of darkness, with three groups of leaves of c. $1 \mathrm{~g}$ each. (c) Coronatine as a virulence factor. Bacteria with coronatine (Pto, COR+) or without coronatine (Pto $\left.\left(\mathrm{Cfa}^{-}\right), \mathrm{COR}-\right)$ were inoculated and their growth measured as in Figure 1. (d) Resistance induced by ethylene. The plants were treated with $1 \mathrm{mM}$ 1-aminocyclopropane-1-carboxylic acid (ACC) or a mock treatment, and then Pto was inoculated and measured as in Figure 1. etr 1 is a negative control of resistance induced by ethylene. One asterisk means a significant difference with $\mathrm{P}<0.05$, and two asterisks means $\mathrm{P}<0.01$ (Student's test of one tail).

direct or indirect negative interference of NPR1 with the BOPs would be favored stoichiometrically. Once the pathogen was inoculated, the levels of SA would rise and in a wild type plant NPR1 is degraded as part of the signaling process [48]. In an nprl background, this signaling would not be transmitted and perhaps the npr1 proteins would be able to interfere longer in RIM. This would explain the behavior of NPR1-HBD in npr1-1 (v 2b); NPR1-HBD in the cytoplasm did not complement npr1-1 in the RIM phenotype, but when DEX was applied there was complementation of the phenotype. Likely, when no DEX was present npr1-1 would somehow interfere with the function of the BOPs. When DEX was present, the presence of NPR1-HBD in the nucleus would trigger the degradation of both NPR1-HBD and npr1-1. If npr1-1 was degraded, the BOPs would function normally.
There was no evidence for a direct interaction in yeast, since the presence of NPR1 or mutated versions of this protein did not interfere in the interaction between BOP1 and BOP2 in a consistent manner with the phenotype (Figure 8a). A first alternative was that the interference of the RIM- alleles would occur with the BOPs without affecting the interaction between the BOPs. A second alternative would be that the RIM- alleles would interfere with other proteins that normally interact with the BOPs. In both cases there is a family of proteins that interacts with both NPR1 and the BOPs, the transcription factors TGAs [49], with -again- functional redundancy (Figure 8b). Two out of three RIM- alleles enhanced or stabilized the BOPs-TGAs interaction, while two out of three RIM+ alleles did not (Figure 8c and data not shown). It was clear that the npr1 mutated proteins had an unpredicted effect on the BOPs-TGAs 

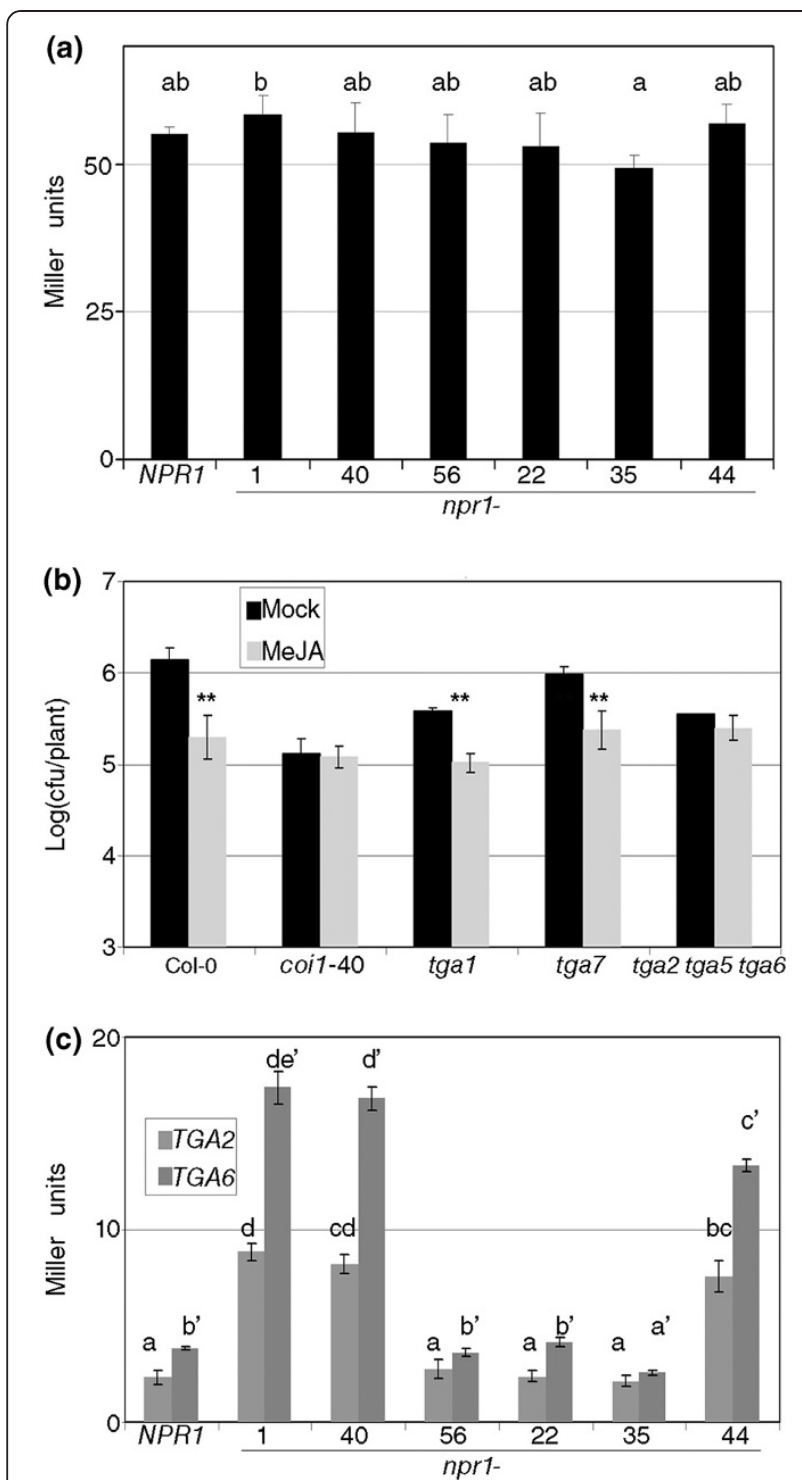

Figure 8 The TGA family plays an important role in resistance induced by MeJA. (a) Interaction of BOP1 and BOP2 in the yeast two hybrid system in presence of several nprl alleles cloned in a third plasmid. NPR1 is included as control, the next three alleles do not respond to MeJA in defense, and the remaining three respond as wild type. The interaction is measured in Miller Units [55]. (b) Null alleles of several TGAs alleles were analyzed as in Figure 1. tga2,5,6 stands for the triple tga 2 tga5 tga6. One asterisk means a significant difference with $P<0.05$, and two asterisks means $P<0.01$ (Student's test of one tail). (c) Interaction between BOP1 and TGA2, and TGA6 in the yeast two hybrid system in the presence of the same npr 1 alleles of (a). Means with the same letter are not significantly different (Fisher's LSD test, $P<0.05$ )

interaction, but the yeast experiments did not produce an absolute answer about the role of npr1 proteins in RIM. We speculate that in planta, all the RIM- alleles enhance the interaction between the BOPs and the TGAs, titering out the TGAs and thus rendering them unable to fulfill their function of triggering defense. On the other hand, the RIM+ alleles (including the null alleles), and NPR1 would not affect the interaction either way. Since in the yeast assays two out of three alleles worked as proposed in either way, it may be possible that a factor(s) is present in the plant that is not in yeast, or perhaps the fact that there are ten TGAs [49], and that NPR1 is expressed between 3 and 18 times more than $B O P 1+B O P 2$ (Additional file 1) could explain this difference. If this hypothesis were to be true, it will definitively explain the role of npr1 in RIM.

\section{Conclusions}

In sum, we have shown that, in wild type conditions, the $B O P s$ and the TGAs (but not NPR1) are required for the resistance triggered by methyl jasmonate against Pto. We propose that the phenotype of the npr1 RIM- alleles is caused by their interference between BOPs and TGAs.

\section{Methods}

Plant growth and inoculation

Arabidopsis thaliana (L.) Heynh. was sown and grown as described [23] in controlled environment rooms with days of $8 \mathrm{~h}$ at $21^{\circ} \mathrm{C}, 150 \mu \mathrm{mol} \mathrm{m} \mathrm{s}^{-2}$, and nights of $16 \mathrm{~h}$ at $19^{\circ} \mathrm{C}$. Treatments, inoculations, and sampling started 30 minutes after the initiation of the artificial day to ensure reproducibility. The following genotypes were used: npr1-1 and npr1-3 [27]; npr2, npr1-20 to npr1-71, and combinations of npr1-70 with other genotypes [11]; 35SCaMVp:NPR1HBD [15]; sid2 [30]; eds5 [29]; NahG [28]; npr3 and npr4 [7]; bop1-3 and bop2-1 [21]; coi1-40 (Dobón, Wulff, Canet and Tornero, to be published elsewhere); kant6, pan1-1 to pan1-3, tga1, and tga7 [50]; 35S:BOP1 and 35S:BOP2 [33]; etr1-3 [39]; tga2 tga5 tga6 [41]. Pseudomonas syringae pv. tomato DC3000 (Pto) was grown, inoculated and measured as described [51]. Briefly, plants of 14 days were inoculated by spray with Pto at $\mathrm{OD}_{600}=0.1$ with $0.02 \%$ Silwet L-77 (Crompton Europe Ltd, Evesham, UK). Three days later, the amount of colony forming units (cfu) per plant was quantified and represented in a logarithmic scale. When indicated, a strain of Pto lacking coronatine was used (Pto(cfa ${ }^{-}$), [37]). For all the experiments, at least three independent treatments were performed (three independent sets of plants sown and treated on different dates).

\section{Expression in planta and in yeast}

NPR1 and six alleles of this gene were cloned in pDONR222 or pDONR221 (Invitrogen, Barcelona, Spain) and then transferred to pMDC43 [52] for expression in planta with GFP and to pARC352 [53] for expression in yeast. Similarly, BOP1, BOP2, TGA2, TGA5, and TGA6, were cloned and then transferred to pDEST22 and pDEST32 (Invitrogen) for expression in yeast. Yeast n-hybrid analyses were done as described 
[54], and the interactions were quantified as described [55]. $N$. benthamiana leaf tissue was mounted in water under a coverslip 4 days after infiltration with Agrobacterium tumefaciens containing the constructs. All imaging was conducted with a Leica TCS SL confocal laser scanning microscope (Leica, Barcelona, Spain) using an HCX PL APO CS 40X/1.25 oil objective to study the subcellular localization of the fluorescence-tagged proteins. Green fluorescent protein was visualized by 488 $\mathrm{nm}$ excitation with an Ar laser, and its emissions were examined with a band-pass filter for 500 to $530 \mathrm{~nm}$. The primers used are included as Additional file 1. Primers and chemical products were purchased from SIGMA (St. Louis, MO, USA) unless otherwise is stated. For the construction of amiRNA(BOP1-BOP2), the plasmid pRS300 was modified [32], cloned in pGW14 [56], and plants were transformed as described [57].

\section{Chemical treatments}

To measure the effect in Pto growth $100 \mu \mathrm{M}$ methyl jasmonate (MeJA) in 0.1\% DMSO and 0.02\% Silwet L-77 (Crompton Europe Ltd) was applied by spray one day before the pathogen inoculation [25]. Dexamethasone was applied at $2 \mu \mathrm{M}$ diluted in water from a stock of $20 \mathrm{mM}$ in EtOH. 1-Aminocyclo- propane-1-carboxylic acid (ACC) was sprayed at $1 \mathrm{mM}$ in water with $0.02 \%$ Silwet L-77.

\section{In vitro growth}

For in vitro culture, plants were grown in Johnson's media [58] with $1 \mathrm{mM} \mathrm{KH_{2 }} \mathrm{PO}_{4}$. When indicated, the plates were supplemented with $50 \mu \mathrm{M}$ MeJA. The length of the roots was measured with ImageJ software [59]. Senescence induced by MeJA was measured as described [36].

\section{RT-qPCR}

Total RNA from 3-week-old (Figure 5c) or 6-week-old plants (Figure 6b) was extracted with Trizol (Invitrogen), following the manufacturer's instructions. cDNA was synthesized with RevertAid ${ }^{\text {th }}$ First Strand cDNA Synthesis Kit (Fermentas, Madrid, Spain), and the quantitative PCR performed with LuminoCt Sybr Green qPCR Ready Mix (SIGMA) in a 7000 RT-PCR Systems machine (Applied Biosystems, Madrid, Spain), following the manufacturer's instructions. For each measurement three biological replicates were done. The obtained values were referred to the geometric average of three reference genes (At3G18780, At1G49240, and At5G60390), as described [60], and normalized, being the value of Col-0 in mock equal to one. The list of primers used is provided in Additional file 1.

\section{Additional files}

Additional file 1: Figure S1. Localization of cloned npr1 alleles mentioned in the text. Figure S2. NPR1HBD treated with DEX is more sensitive to BTH. Figure S3. Expression levels of NPR1 paralogs. Figure S4. Response of bop1 bop2 to SA and BTH. Table S1. List of primers used.

Competing interests

The authors declare that they do not have any competing interest.

\section{Authors' contributions}

JVC, AD, and JF performed the experiments, analyzed the data and revised the paper. PT designed the research, analyzed the data and wrote the paper. All authors read and approved the final manuscript.

\section{Acknowledgements}

This work was supported by the "Ministerio de Economía y Competitividad" (MINECO) of Spain (grant BIO201018896 to PT, a JAE-CSIC Fellowship to JVC and a FPI-MINECO to AD) and "Generalitat Valenciana" of Spain (grant ACOMP/2012/105 to PT). Thanks to Dr. Xinnian Dong for NPR1 overexpression lines and to Dr. Ove Nilsson for BOPs overexpression lines. We appreciate the opinions and generous help of Drs. Vicente Ramirez, Pablo Vera, and Shelley Hepworth about the manuscript.

\section{Author details}

${ }^{1}$ Current address: Department of Crop Genetics, The John Innes Centre, Norwich Research Park, Norwich, NR4 7UH, UK. ${ }^{2}$ Current address: Department of Economics and Management of Chemical and Food Industry, Faculty of Chemical Engineering, Institute of Chemical Technology Prague (ICT), Technická 5, Prague 6, Dejvice 166 28, Czech Republic. ${ }^{3}$ Instituto de Biología Molecular y Celular de Plantas (IBMCP), Universidad Politécnica de Valencia (UPV)-Consejo Superior de Investigaciones Científicas (CSIC), Ciudad Politécnica de la Innovación (CPI), Ed. 8E; C/ Ingeniero Fausto Elio s/n, Valencia 46022, Spain.

Received: 8 June 2012 Accepted: 24 October 2012

Published: 2 November 2012

\section{References}

1. Vlot AC, Dempsey DMA, Klessig DF: Salicylic Acid, a Multifaceted Hormone to Combat Disease. Ann Rev Phytopathology 2009, 47(1):177-206.

2. Katagiri F, Thilmony S, He SY: The Arabidopsis thaliana-Pseudomonas syringae Interaction. In The Arabidopsis Book. Rockville: American Society of Plant Biologists; 2002. doi:10.1199/tab.0111. http://www.aspb.org/ publications/arabidopsis/.

3. Ross AF: Systemic acquired resistance induced by localized virus infections in plants. Virology 1961, 14:340-358.

4. Lopez MA, Bannenberg G, Castresana C: Controlling hormone signaling is a plant and pathogen challenge for growth and survival. Curr Opin Plant Biol 2008, 11(4):420-427.

5. Browse J: Jasmonate Passes Muster: A Receptor and Targets for the Defense Hormone. Annu Rev Plant Biol 2009, 60(1):183-205.

6. Dong X: NPR1, all things considered. Curr Opin Plant Biol 2004, 7(5):547-552.

7. Zhang Y, Cheng YT, Qu N, Zhao Q, Bi D, Li X: Negative regulation of defense responses in Arabidopsis by two NPR1 paralogs. Plant J 2006, 48(5):647-656.

8. Ha CM, Kim GT, Kim BC, Jun JH, Soh MS, Ueno Y, Machida Y, Tsukaya H, Nam HG: The BLADE-ON-PETIOLE 1 gene controls leaf pattern formation through the modulation of meristematic activity in Arabidopsis. Development 2003, 130(1):161-172.

9. Liu G, Holub EB, Alonso JM, Ecker JR, Fobert PR: An Arabidopsis NPR1-like gene, NPR4, is required for disease resistance. Plant J 2005, 41(2):304-318.

10. Fu ZQ, Yan S, Saleh A, Wang W, Ruble J, Oka N, Mohan R, Spoel SH, Tada Y, Zheng N, et al: NPR3 and NPR4 are receptors for the immune signal salicylic acid in plants. Nature 2012, 486(7402):228-232.

11. Canet JV, Dobón A, Roig A, Tornero P: Structure-Function Analysis of npr1 Alleles in Arabidopsis Reveals a Role for its Paralogs in the Perception of Salicylic Acid. Plant Cell Environ 2010, 33:1911-1922. 
12. Zhang $Y$, Fan W, Kinkema M, Li X, Dong X: Interaction of NPR1 with basic leucine zipper protein transcription factors that bind sequences required for salicylic acid induction of the PR-1 gene. Proc Natl Acad Sci U S A 1999, 96(11):6523-6528.

13. Pieterse CM, van Wees SC, van Pelt JA, Knoester M, Laan R, Gerrits $H$, Weisbeek PJ, van Loon LC: A novel signaling pathway controlling induced systemic resistance in Arabidopsis. Plant Cell 1998, 10(9):1571-1580.

14. Ton J, De Vos M, Robben C, Buchala A, Metraux JP, Van Loon LC, Pieterse CM: Characterization of Arabidopsis enhanced disease susceptibility mutants that are affected in systemically induced resistance. Plant J 2002, 29(1):11-21.

15. Kinkema M, Fan W, Dong X: Nuclear localization of NPR1 is required for activation of PR gene expression. Plant Cell 2000, 12(12):2339-2350.

16. Spoel SH, Koornneef A, Claessens SM, Korzelius JP, Van Pelt JA, Mueller MJ, Buchala AJ, Metraux JP, Brown R, Kazan K, et al: NPR1 modulates cross-talk between salicylate- and jasmonate-dependent defense pathways through a novel function in the cytosol. Plant Cell 2003, 15(3):760-770.

17. Glazebrook J, Chen W, Estes B, Chang HS, Nawrath C, Metraux JP, Zhu T, Katagiri F: Topology of the network integrating salicylate and jasmonate signal transduction derived from global expression phenotyping. Plant J 2003, 34(2):217-228.

18. Johansson A, Staal J, Dixelius C: Early responses in the ArabidopsisVerticillium longisporum pathosystem are dependent on NDR1, JA- and ET-associated signals via cytosolic NPR1 and RFO1. Mol Plant Microbe Interact 2006, 19(9):958-969.

19. Leon-Reyes A, Spoel SH, De Lange ES, Abe H, Kobayashi M, Tsuda S, Millenaar FF, Welschen RA, Ritsema T, Pieterse CM: Ethylene modulates the role of NONEXPRESSOR OF PATHOGENESIS-RELATED GENES1 in cross talk between salicylate and jasmonate signaling. Plant Physiol 2009, 149(4):1797-1809.

20. Ramirez V, Van der Ent S, Garcia-Andrade J, Coego A, Pieterse CM, Vera P: OCP3 is an important modulator of NPR1-mediated jasmonic aciddependent induced defenses in Arabidopsis. BMC Plant Biol 2010, 10:199.

21. Hepworth SR, Zhang Y, McKim S, Li X, Haughn GW: BLADE-ON-PETIOLEdependent signaling controls leaf and floral patterning in Arabidopsis. Plant Cell 2005, 17(5):1434-1448.

22. Ha CM, Jun JH, Nam HG, Fletcher JC: BLADE-ON-PETIOLE1 encodes a BTB/ $\mathrm{POZ}$ domain protein required for leaf morphogenesis in Arabidopsis thaliana. Plant Cell Physiol 2004, 45(10):1361-1370.

23. Canet JV, Dobón A, Ibáñez F, Perales L, Tornero P: Resistance and biomass in Arabidopsis: a new model for Salicylic Acid perception. Plant Biotech J 2010, 8:126-141.

24. Cao H, Bowling SA, Gordon AS, Dong X: Characterization of an Arabidopsis mutant that is nonresponsive to inducers of systemic acquired resistance. Plant Cell 1994, 6(11):1583-1592.

25. Dobón A, Canet JV, Perales L, Tornero P: Quantitative genetic analysis of salicylic acid perception in Arabidopsis. Planta 2011, 234:671-684.

26. Zhang X, Chen S, Mou Z: Nuclear localization of NPR1 is required for regulation of salicylate tolerance, isochorismate synthase 1 expression and salicylate accumulation in Arabidopsis. J Plant Physio/ 2010, 167(2):144-148.

27. Cao H, Glazebrook J, Clarke JD, Volko S, Dong X: The Arabidopsis NPR1 gene that controls systemic acquired resistance encodes a novel protein containing ankyrin repeats. Cell 1997, 88(1):57-63.

28. Lawton K, Weymann K, Friedrich L, Vernooij B, Uknes S, Ryals J: Systemic acquired resistance in Arabidopsis requires salicylic acid but not ethylene. Mol Plant Microbe Interact 1995, 8(6):863-870.

29. Nawrath C, Heck S, Parinthawong N, Metraux JP: EDS5, an essential component of salicylic acid-dependent signaling for disease resistance in Arabidopsis, is a member of the MATE transporter family. Plant Cell 2002, 14(1):275-286.

30. Wildermuth MC, Dewdney J, Wu G, Ausubel FM: Isochorismate synthase is required to synthesize salicylic acid for plant defence. Nature 2001, 414(6863):562-565.

31. Khan M, Xu M, Murmu J, Tabb P, Liu Y, Storey K, McKim SM, Douglas CJ, Hepworth SR: Antagonistic interaction of BLADE-ON-PETIOLE1 and 2 with BREVIPEDICELLUS and PENNYWISE regulates Arabidopsis inflorescence architecture. Plant Physiol 2011, 158(2):946-960.

32. Schwab R, Ossowski S, Riester M, Warthmann N, Weigel D: Highly specific gene silencing by artificial microRNAs in Arabidopsis. Plant Cell 2006, 18(5):1121-1133.
33. Norberg M, Holmlund M, Nilsson O: The BLADE ON PETIOLE genes act redundantly to control the growth and development of lateral organs. Development 2005, 132(9):2203-2213.

34. Xie D-X, Feys BF, James S, Nieto-Rostro M, Turner JG: COI1: An Arabidopsis gene required for jasmonate-regulated defense and fertility. Science 1998, 280:1091-1094.

35. Dombrecht B, Xue GP, Sprague SJ, Kirkegaard JA, Ross JJ, Reid JB, Fitt GP, Sewelam N, Schenk PM, Manners JM, et al: MYC2 Differentially Modulates Diverse Jasmonate-Dependent Functions in Arabidopsis. Plant Cell 2007, 19(7):2225-2245

36. He Y, Fukushige H, Hildebrand DF, Gan S: Evidence supporting a role of jasmonic acid in Arabidopsis leaf senescence. Plant Physiol 2002, 128(3):876-884.

37. Mittal S, Davis KR: Role of the phytotoxin Coronatine in the infection of Arabidopsis thaliana by Pseudomonas syringae pv. tomato. Mol Plant Microbe Interact 1995, 8(1):165-171.

38. Ton J, Mauch-Mani B: Beta-amino-butyric acid-induced resistance against necrotrophic pathogens is based on ABA-dependent priming for callose. Plant J 2004, 38(1):119-130.

39. Chang C, Kwok SF, Bleecker AB, Meyerowitz EM: Arabidopsis ethyleneresponse gene ETR1: Similarity of product to two-component regulators. Science 1993, 262:539-544.

40. Jun $\mathrm{JH}, \mathrm{Ha}$ CM, Fletcher JC: BLADE-ON-PETIOLE1 coordinates organ determinacy and axial polarity in arabidopsis by directly activating ASYMMETRIC LEAVES2. Plant Cell 2010, 22(1):62-76.

41. Zhang $Y$, Tessaro MJ, Lassner M, Li X: Knockout analysis of Arabidopsis transcription factors TGA2, TGA5, and TGA6 reveals their redundant and essential roles in systemic acquired resistance. Plant Cell 2003, 15(11):2647-2653.

42. Delaney TP, Friedrich L, Ryals JA: Arabidopsis signal transduction mutant defective in chemically and biologically induced disease resistance. Proc Natl Acad Sci U S A 1995, 92(14):6602-6606.

43. Ha CM, Jun JH, Nam HG, Fletcher JC: BLADE-ON-PETIOLE 1 and 2 control Arabidopsis lateral organ fate through regulation of LOB domain and adaxial-abaxial polarity genes. Plant Cell 2007, 19(6):1809-1825.

44. Xu M, Hu T, McKim SM, Murmu J, Haughn GW, Hepworth SR: Arabidopsis BLADE-ON-PETIOLE1 and 2 promote floral meristem fate and determinacy in a previously undefined pathway targeting APETALA1 and AGAMOUS-LIKE24. Plant J 2010, 63(6):974-989.

45. McKim SM, Stenvik GE, Butenko MA, Kristiansen W, Cho SK, Hepworth SR, Aalen RB, Haughn GW: The BLADE-ON-PETIOLE genes are essential for abscission zone formation in Arabidopsis. Development 2008, 135(8):1537-1546.

46. Ha CM, Jun JH, Fletcher JC: Control of Arabidopsis leaf morphogenesis through regulation of the YABBY and KNOX families of transcription factors. Genetics 2010, 186(1):197-206.

47. Stein E, Molitor A, Kogel KH, Waller F: Systemic resistance in Arabidopsis conferred by the mycorrhizal fungus Piriformospora indica requires jasmonic acid signaling and the cytoplasmic function of NPR1. Plant Cell Physiol 2008, 49(11):1747-1751.

48. Spoel SH, Mou Z, Tada Y, Spivey NW, Genschik P, Dong X: Proteasomemediated turnover of the transcription coactivator NPR1 plays dual roles in regulating plant immunity. Cell 2009, 137(5):860-872.

49. Jakoby M, Weisshaar B, Droge-Laser W, Vicente-Carbajosa J, Tiedemann J, Kroj T, Parcy F: bZIP transcription factors in Arabidopsis. Trends Plant Sci 2002, 7(3):106-111.

50. Alonso JM, Stepanova AN, Leisse TJ, Kim CJ, Chen H, Shinn P, Stevenson DK, Zimmerman J, Barajas P, Cheuk R, et al: Genome-wide insertional mutagenesis of Arabidopsis thaliana. Science 2003, 301(5633):653-657.

51. Tornero P, Dangl JL: A high throughput method for quantifying growth of phytopathogenic bacteria in Arabidopsis thaliana. Plant J 2001, 28(4):475-481.

52. Curtis MD, Grossniklaus U: A gateway cloning vector set for highthroughput functional analysis of genes in planta. Plant Physiol 2003, 133(2):462-469.

53. Ciannamea S, Kaufmann K, Frau M, Tonaco IA, Petersen K, Nielsen KK, Angenent GC, Immink RG: Protein interactions of MADS box transcription factors involved in flowering in Lolium perenne. J Exp Bot 2006, 57(13):3419-3431

54. Vidal M, Legrain P: Yeast forward and reverse ' $n$ '-hybrid systems. Nucleic Acids Res 1999, 27(4):919-929. 
55. Miller JH: Experiments in Molecular Genetics. Cold Spring Harbor, New York: Cold Spring Harbor Laboratory; 1972.

56. Nakagawa T, Kurose T, Hino T, Tanaka K, Kawamukai M, Niwa Y, Toyooka K, Matsuoka $K$, Jinbo T, Kimura T: Development of series of gateway binary vectors, pGWBs, for realizing efficient construction of fusion genes for plant transformation. J Biosci Bioeng 2007, 104(1):34-41.

57. Clough SJ, Bent AF: Floral dip: a simplified method for Agrobacteriummediated transformation of Arabidopsis thaliana. Plant J 1998, 16(6):735-743.

58. Johnson CM, Stout PR, Broyer TC, Carlton AB: Comparative chlorine requirements of different plant species. Plant Soil 1957, 8:337-353.

59. Abramoff MD, Magalhaes PJ, Ram SJ: Image Processing with ImageJ. Biophotonics International 2004, 11(7):36-42.

60. Vandesompele J, De Preter K, Pattyn F, Poppe B, Van Roy N, De Paepe A, Speleman F: Accurate normalization of real-time quantitative RT-PCR data by geometric averaging of multiple internal control genes. Genome Biol 2002, 3(7). RESEARCH0034.

doi:10.1186/1471-2229-12-199

Cite this article as: Canet et al.: The BLADE-ON-PETIOLE genes of Arabidopsis are essential for resistance induced by methyl jasmonate. BMC Plant Biology 2012 12:199.

\section{Submit your next manuscript to BioMed Central and take full advantage of:}

- Convenient online submission

- Thorough peer review

- No space constraints or color figure charges

- Immediate publication on acceptance

- Inclusion in PubMed, CAS, Scopus and Google Scholar

- Research which is freely available for redistribution 